\title{
BOUNDED, CONSERVATIVE, LINEAR OPERATORS AND THE MAXIMAL GROUP. II
}

\author{
E. P. KELLY, JR. AND D. A. HOGAN
}

\begin{abstract}
Let $V$ denote an infinite dimensional Banach space over the complex field, $B[V]$ the bounded linear operators on $V$ and $F$ a closed subspace of $V$. An element of $\mathscr{T}_{F}=\{T \mid T \in B[V]$, $T(F) \subseteq F\}$ is called a conservative operator. Some sufficient conditions for $T \in \mathscr{T}_{F}$ to be in the boundary, $\mathscr{B}$, of the maximal group, $\mathscr{M}$, of invertible elements are determined. For example, if $T \in \mathscr{T}_{F}$ is such that (i) $V$ is the topological direct sum of $\mathscr{R}(T)$ and $N(T) \neq$ $\{\theta\}$, (ii) $T$ is an automorphism on $\mathscr{R}(T) \cap F$, then $T \in \mathscr{B}$. Also, the complement of the closure of $\mathscr{M}$ is discussed. This is an extension of another paper by the same authors [6].
\end{abstract}

1. Introduction. In [6], a bounded, linear operator, $T$, on a Banach space, $V$, which contains a complete subspace which is invariant under $T$ was called a conservative operator. In particular, the complete subspace was taken to be the domain of a linear functional. Most of the results in [6] do not depend on the complete subspace being the domain of a linear functional. (For clarification, in the example (1) [6, p. 195], $n$ is fixed and $B[c]$ should be $B[V]$.) Hence, in this paper a conservative operator means a bounded, linear operator on an infinite dimensional Banach space which leaves a closed subspace, $F$, of $V$ invariant. Let $B[V]$ denote the Banach algebra of bounded linear operators on $V$ and $\mathscr{T}_{F}=\{T \mid T \in B[V], T(F) \subseteq$ $F\}$. It is readily seen that $\mathscr{T}_{F}$ is a Banach subalgebra of $B[V]$ and hence the following facts are available:

(i) $\mathscr{M}=\left\{T \mid T^{-1}, T \in \mathscr{T}_{F}\right\}$, the maximal group of $\mathscr{T}_{F}$, is open,

(ii) $T \in \mathscr{M}$ if and only if $T^{-1} \in B[V]$ and $T(F)=F$,

(iii) the boundary, $\mathscr{B}$, of $\mathscr{M}$ is a multiplicative semigroup,

(iv) if $T_{1} \in \mathscr{M}$ and $T_{2} \in \mathscr{B}$, then $T_{1} T_{2}$ and $T_{2} T_{1} \in \mathscr{B}$.

Apparently necessary and sufficient conditions for $T \in \mathscr{T}_{F}$ to be in $\mathscr{B}$ are not known. However, they are known in the case of a weakly closed, selfadjoint operator algebra on a Hilbert space [5]. It is known [8] that $\mathscr{B}$ is contained in the set of $T \in \mathscr{T}_{F}$ which are both left and right topological divisors of zero.

Received by the editors December 21, 1971 and, in revised form, June 15, 1972.

AMS (MOS) subject classifications (1970). Primary 46L20, 47D10; Secondary 40C05.

Key words and phrases. Banach algebra, maximal group, boundary of maximal group, bounded operator, conservative operator, projection, quasi-compact operator.

(c) American Mathematical Society 1973 
Herein, $\mathscr{R}(T)$ will denote the range space of $T, N(T)$ will denote the null space of $T$ and $I$ will denote the identity operator. The results of [6] carry over to the Banach algebra $\mathscr{T}_{F}$ and this paper extends the work presented in [6].

2. Sufficient conditions for $T \in \mathscr{T}_{F}$ to be in $\mathscr{B}$. In [7], Rhoades determined that certain classical triangular matrix summability operators are in the boundary of the maximal group of conservative, triangular operators. In [6] two questions raised in [7] were answered, by examples of matrices whose products are compact operators on $V=m$, the space of bounded complex sequences. Actually the following classes of operators in $\mathscr{T}_{F}$ are subsets of $\mathscr{B}$ :

(i) projections, other than $I$,

(ii) quasi-compact operators,

(iii) quasi-nilpotent operators.

The facts depend on $V$ being infinite dimensional and follow from spectral properties of these types of operators ([4], [8]). It should be noted that the collection of compact operators, which form a closed two-sided ideal of $\mathscr{T}_{F}$, is contained in $\mathscr{B}$.

THEOREM 1. If $T \in \mathscr{T}_{F}$ is such that

(i) $V$ is the topological direct sum of $\mathscr{R}(T)$ and $N(T) \neq\{\theta\}$,

(ii) $T$ is an automorphism on $\mathscr{R}(T) \cap F$, then $T \in \mathscr{B}$.

Proof. Let $P$ denote the projection of $V$ onto $\mathscr{R}(T)$ along $N(T)$. If $y+z=x \in F$, where $y \in \mathscr{R}(T), z \in N(T)$, then $P(x)=y$. Now $T(x)=T(y) \in$ $F$ and from (i) there is an $\alpha \in \mathscr{R}(T)$ such that $T(\alpha)=y$. Then $T(y) \in F \cap$ $\mathscr{R}(T)$ and (ii) implies $\alpha \in F \cap \mathscr{R}(T)$. Hence, $y=T(\alpha) \in F \cap \mathscr{R}(T)$. Thus, $P \in \mathscr{T}_{F}$. Moreover, $M=T+I-P \in \mathscr{T}_{F}$. It can be verified that $M$ is oneto-one and onto $V$.

Now $y+z=x \in F$ where $y \in \mathscr{R}(T) \cap F, z \in N(T)$. Then $x=M(\alpha+z)$, where $\alpha \in F \cap \mathscr{R}(T)$ and $T(\alpha)=y$. Thus $M^{-1}(F)=F$. Hence, $M \in \mathscr{M}$.

Since $M P=(T+I-P) P=T, M \in \mathscr{M}, P \in \mathscr{B}$, it follows that $T \in \mathscr{B}$. The following corollary is readily verified.

CoRollary 1. If $T \in \mathscr{T}_{F}$ is such that

(i) $V$ is the topological direct sum of $\mathscr{R}(T)$ and $N(T) \neq\{\theta\}$,

(ii) $T^{-1}(F)=F$, then $T \in \mathscr{B}$.

It should be noted that Corollary 1 is essentially Lemma 2 of [6]. (For clarification, in Lemma 2 [6], $V=S \oplus T(V)$ indicates the direct sum of $S$ and $T(V)$ and (ii) hypothesizes the existence of a bounded projection of $V$ 
onto $S$, insuring that the projection $P$ in the proof, the projection of $V$ onto $S$ along $T(V)$, is bounded. Also, in the sentence preceding Lemma 2 [6], $T \in V$ should read $T \in B[V]$.) The hypotheses of Corollary 1 seem rather strong since (i) may not be satisfied for finite dimensional $V$. However, (i) is satisfied if $T \neq I$ is a bounded projection on $V$. It is readily seen that (ii) is not a necessary condition for $T$ to be in $\mathscr{B}$, since it is not satisfied by the null operator $Z$ and $Z \in \mathscr{B}$.

In the case where $V=m$ and $F=c$, the space of convergent sequences, (ii) of Corollary 1 says that $T$ limits no bounded divergent sequences. Now, Whitley ([9], [12]) showed that a matrix $T \in B[m]$ is such that $T^{-1}(c)=c$ if and only if it is range closed and has a finite dimensional null space. Hence, if a matrix $T \in B[\mathrm{~m}]$ satisfies (i) of Corollary 1 and has a finite dimensional null space, then (ii) of Corollary 1 is satisfied and $T \in \mathscr{B}$. There are examples [6] of matrices in $B[\mathrm{~m}]$ which have closed range and a finite dimensional null space which are not in $\mathscr{B}$ nor in $\mathscr{M}$. There are matrices $T$ in $B[m]$ for which $T \in \mathscr{B}$ and $c$ is a proper subspace of $T^{-1}(c)$ ([2], [3], [7]). Thus it seems that in this setting a stronger condition than (ii) of Corollary 1 is needed for a set of necessary and sufficient conditions for $T$ to be in $\mathscr{B}$.

Since Theorem 1 provides weaker conditions than Lemma 2 [6], Theorems 2 and 3 of [6] are now stated, for completeness, in the setting of this paper.

THEOREM 2. Let $T_{1} \in \mathscr{B}$ be one-to-one. For $T_{2} \in \mathscr{T}_{F}$, if

(i) $T_{2}(V) \subseteq T_{1}(V)$,

(ii) $T_{1}^{-1} T_{2}$ satisfies (i) of Theorem 1 ,

(iii) $T_{2}(F)=T_{1}(F)$,

(iv) $T_{2}^{-1}(F)=F$,

then $T_{2} \in \mathscr{B}$.

THEOREM 3. Let $T_{1} \in \mathscr{B}$ be one-to-one. For $T_{2} \in \mathscr{T}_{F}$, if

(i) $T_{2}(V)=T_{1}(V)$,

(ii) $T_{2}$ is one-to-one,

(iii) $T_{2}(F)=T_{1}(F)$, then $T_{2} \in \mathscr{B}$.

It should be noted that $T_{1}$ in the above theorems, being one-to-one and in $\mathscr{B}$, implies that $T_{1}$ is a one-to-one, two-sided topological divisor of zero and hence cannot be onto [10, Theorem 5].

In [3], certain conservative matrices in $B[m]$ are expressed as $I+P$, where the range space of $P$ is finite dimensional. The following theorem shows that these matrices are in $\overline{\mathscr{M}}$, the closure of $\mathscr{M}$.

TheOREM 4. If $T_{1} \in \mathscr{T}_{F}$ is compact and $T_{2} \in \overline{\mathcal{M}}$, then $T_{1}+T_{2} \in \bar{M}$. 
Proof. It is sufficient to prove that $T_{1}+T_{2} \in \overline{\mathscr{M}}$, if $T_{2} \in \mathscr{M}$. Hence, if $T_{2} \in \mathscr{M}$, then $T_{2}^{-1} \in \mathscr{M}$. Also $T_{2}^{-1} T_{1}$ is a compact operator. Thus $I+$ $T_{2}^{-1} T_{1} \in \overline{\mathscr{M}}$ and so $T_{2}+T_{1}=T_{2}\left(I+T_{2}^{-1} T_{1}\right) \in \overline{\mathscr{M}}$.

There are several interesting geometrical facts about $\mathscr{B}$, such as $\mathscr{B}$ is connected and $\mathscr{B}$ is not convex.

3. The complement of $\overline{\mathscr{M}}$. Let $\mathscr{N}$ denote the complement of $\overline{\mathscr{M}}$. Note that (i) of Theorem 1 implies that $\mathscr{R}(T)$ is closed. However, if $\mathscr{R}(T)$ is closed, it does not follow that $T \in \mathscr{B}$ [11]. Several sufficient conditions for $T$ to be in $\mathscr{N}$ are known, such as if $T \in \mathscr{T}_{F}$ satisfies either of the following:

(i) $T$ is one-to-one and $\mathscr{R}(T)$ is a closed proper subset of $V$,

(ii) $T$ is onto but not one-to-one,

(iii) $T^{-1} \in B[V]$ and $T(F) \neq F$.

Several facts pertaining to products of $T \in \mathscr{N}$ and $T_{1} \in \mathscr{T}_{F}$ are given in [6]. Two other interesting results follow.

Theorem 5. If $T \in \mathscr{N}$ and $T T_{1} \in \mathscr{M}$, then $T_{1} \in \mathscr{N}$.

Proof. Note $T_{1} \notin \mathscr{M}$, for if so, $T T_{1} \in \mathscr{M}$ implies $T \in \mathscr{M}$. Since $T T_{1}=T_{2} \in \mathscr{M}, T\left(T_{1} T_{2}^{-1}\right)=I$ and $T_{1} T_{2}^{-1}$ is in $\mathscr{M}, \mathscr{B}, \mathscr{N}$ if and only if $T_{1}$ is in $\mathscr{M}, \mathscr{B}, \mathscr{N}$. Thus $T_{1} T_{2}^{-1}$ has a left inverse and must be one-to-one. Moreover, $\left(T_{2}^{-1} T\right) T_{1}=I$ implies $T_{1}$ has a left inverse. Thus $T_{1}$ is range closed. Hence, $T_{1} T_{2}^{-1}$ is range closed. Thus $T_{1} T_{2}^{-1} \in \mathscr{N}$, implying $T_{1} \in \mathscr{N}$.

THEOREM 6. If $T \in \mathscr{N}$ and $P$ is a projection such that $N(P)$ is finite dimensional, then TP and PT are in $\mathscr{N}$.

Proof. Now $I-P$ is compact. Hence, $T(I-P)$ is compact. $T P$ is not in $\mathscr{M}$ by Theorem 5. If $T P \in \mathscr{B}$, then by Theorem $4, T P+T(I-P)=T \in \mathscr{B}$, contradicting the hypothesis. Thus, $T P \in \mathscr{N}$. Similarly, $P T \in \mathscr{N}$. When $\mathscr{N} \neq \varnothing$, this theorem shows that there are both left and right divisiors of zero in $\mathscr{N}$. It should be noted that for finite dimensional $V, \mathscr{N}=\varnothing$. It remains unknown if $\mathscr{N}=\varnothing$ implies that $V$ is finite dimensional [8].

4. An application of Theorem 6. Take $F=V$. Suppose $T$ has a closed range and a finite dimensional, nonzero, null space, and $V$ is the topological direct sum of $\mathscr{R}(T)$ and another closed subspace of $V$. Hence, there is a projection, $P_{1}$, and a $T_{1} \in \mathscr{T}_{F}=B[V]$ such that $T=$ $T_{1} P_{1}$, where $T_{1} \in \mathscr{M}$ or $T_{1} \in \mathscr{N}$. To review this factorization of $T$, note that $V=\mathscr{R}(T) \oplus K_{2}$, where $K_{2}$ is a closed complementary subspace of $\mathscr{R}(T)$ such that $N(T)=\left[K_{2} \cap N(T)\right] \oplus[\mathscr{R}(T) \cap N(T)]$. Thus $V=[\mathscr{R}(T) \cap N(T)] \oplus$ $K_{1} \oplus K_{2}$, where $K_{1}$ is a closed complementary subspace of $\mathscr{R}(T) \cap N(T)$ in $\mathscr{R}(T)$. Now, define $T_{1}: V \rightarrow V$ as follows: $T_{1}=T$ on $K_{1} \oplus K_{3}$, where $K_{3}$ is a closed complementary subspace of $K_{2} \cap N(T)$ in $K_{2} ; T_{1}=I$ on $K_{2} \cap N(T)$; 
and $T_{1}=\mathscr{T}$ on $\mathscr{R}(T) \cap N(T)$, where $\mathscr{T}$ is a linear transformation of $\mathscr{R}(T) \cap N(T)$ onto $K_{3}$ or $\mathscr{T}$ is an isomorphism of $\mathscr{R}(T) \cap N(T)$ into $K_{3}$. Take $P_{1}$ to be the projection of $V$ onto $K_{1} \oplus K_{3}$ along $\left[K_{2} \cap N(T)\right] \oplus[\mathscr{R}(T) \cap N(T)]$. Note that $T_{1} \in \mathscr{M}$ if and only if $\mathscr{T}$ is an isomorphism of $\mathscr{R}(T) \cap N(T)$ onto $K_{3}$. Thus, $T_{1} \in \mathscr{M}$ if and only if codimension of $\mathscr{R}(T)$ equals dimension of $N(T)$. Thus by Theorem $6, T \in \mathscr{B}$ if and only if $T_{1} \in \mathscr{M}$. Thus, $T \in \mathscr{B}$ if and only if codimension of $\mathscr{R}(T)$ equals dimension of $N(T)$. In particular, if $T$ is a matrix in $B[m]$ such that $T^{-1}(c)=c$, then $T$ has a closed range and a finite dimensional null space. So, if such a matrix has a nonzero null space, then it belongs to the boundary of the maximal group if and only if codimension of $\mathscr{R}(T)$ equals dimension of $N(T)$.

\section{REFERENCES}

1. I. D. Berg, A Banach algebra criterion for Tauberian theorems, Proc. Amer. Math. Soc. 15 (19.64), 648-652. MR 29 \#2574.

2. - Open sets of conservative matrices, Proc. Amer. Math. Soc. 16 (1965), 719-724. MR 31 \#3762.

3. J. Copping, Mercerian theorems and inverse transformations, Studia Math. 21 (1961/62), 177-194. MR 25 \#3296.

4. N. Dunford and J. T. Schwartz, Linear operators. I: General theory, Pure and Appl. Math., vol. 7, Interscience, New York, 1958. MR 22 \#8302.

5. J. Feldman and R. V. Kadison, The closure of the regular operators in a ring of operators, Proc. Amer. Math. Soc. 15 (1964), 909-916.

6. E. Kelly and D. Hogan, Bounded, conservative, linear operators and the maximal group, Proc. Amer. Math. Soc. 32 (1972), 195-200.

7. B. E. Rhoades, Triangular summability methods and the boundary of the maximal group, Math. Z. 105 (1968), 284-290. MR 37 \#4461.

8. C. E. Rickart, General theory of Banach algebras, University Series in Higher Math., Van Nostrand, Princeton, N.J., 1960. MR 22 \#5903.

9. $R$. Whitley, Conull and other matrices which sum a bounded divergent sequence, Amer. Math. Monthly 74 (1967), 798-801. MR 36 \#3010.

10. A. Wilansky, Topological divisors of zero and Tauberian theorems, Trans. Amer. Math. Soc. 113 (1964), 240-251. MR 29 \#6222.

11. B. Yood, Transformations between Banach spaces in the uniform topology, Ann. of Math. (2) 50 (1949), 486-503. MR 10, 611.

12. D. J. H. Garling and A. Wilansky, On a summability theorem of Berg, Crawford, and Whitley, Proc. Cambridge Philos. Soc. 71 (1972), 495-497.

Department of Mathematics, Louisiana Tech University, Ruston, Louisiana 71270 (Current address of E. P. Kelly, Jr.)

Current address (D. A. Hogan): Department of Mathematics, Southern Illinois University, Carbondale, Illinois 62901 